\title{
St. Gallen/Vienna 2015: A Brief Summary of the Consensus Discussion
}

\author{
Michael Gnant $^{\mathrm{a}} \quad$ Christoph Thomssen $^{\mathrm{b}} \quad$ Nadia Harbeck $^{\mathrm{c}}$ \\ a Department of Surgery and Comprehensive Cancer Center Vienna, Medical University of Vienna, Austria; \\ ${ }^{b}$ Department of Gynecology, Martin-Luther-University, Halle/Saale, Germany; \\ ${ }^{\mathrm{c}}$ Breast Center, Department of Obstetrics and Gynecology, University of Munich, Germany
}

\section{Keywords}

Early breast cancer - Bisphosphonates .

Endocrine therapy · Chemotherapy - Surgery .

Axillary dissection - Radiotherapy - Targeted therapy · Neoadjuvant therapy

\section{Summary}

The 2015 St. Gallen Consensus Conference on early breast cancer took place in Vienna, Austria, for the first time. After 3 days of high-level presentations by international panel members of clinical trials having been reported recently in the field, the traditional Saturday voting tried to translate the assembled knowledge into clinical treatment recommendations intended to guide clinical practice of breast cancer care for the 'average' patient. This report summarizes the results of the 2015 international panel voting procedures with respect to locoregional and endocrine treatment, chemotherapy, targeted therapy, as well as adjuvant bisphosphonate use. This report is not aimed to replace the official St. Gallen consensus publication - some recommendations may even be altered in the final paper - but should serve as a preliminary rapid report of this important meeting.

\section{Introduction}

The St. Gallen Consensus Conference 2015 (March 18-11), held for the first time in Vienna, Austria, and now chaired by 7 cochairs, focused again on therapy recommendations for early breast cancer which are based on evidence as well as clinical expertise of the international faculty of 22 countries from Europe and the USA but also Asia-Pacific (table 1). The panel openly disclosed any potential conflict of interest (COI) (www.oncoconferences.ch); the COI committee was once more chaired by Harold Burstein (Boston, USA). It was recognized as being unavoidable that individual panel members have financial relationships with commercial organizations engaged in research, innovation, and education. None of the declared conflicts were judged to substantially impact the voting procedure and warrant exclusion of a panel member. However, members with a specific COI were asked to refrain from voting at certain questions.

The motto of this year's panel discussions and voting was: 'Tailoring therapy: towards precision treatment of patients with early breast cancer'. About 3,500 participants from 135 countries saw 3 days of high-level educational lectures addressing local and systemic therapy as well as imaging issues. Interestingly, and against contrary trends of other disease-specific conferences, the number of participants increased compared to the previous meeting - likely also a consequence of the successful move to logistically easier to reach Vienna. Again, a large number of participants from overseas, particularly China, Japan, and the USA, attended and made the meeting the probably most important international breast cancer meeting on European soil from a global perspective.

The Saturday morning consensus panel was again co-chaired by Aaron Goldhirsch (CH/I) and Eric Winer (USA), who also moderated the discussion and voting. This time, more than 170 questions had been developed and exchanged by panelists upfront, which reduced debate and put some time pressure on the panel in order to agree on solutions suitable for breast cancer care around the world. It was again stated that clinical trials provide evidence that shows whether one treatment is better than another and help to estimate an average of outcome improvement, but not how to treat a given individual patient. The primary consideration on which the panel's voting decisions were based upon was to provide

\section{KARGER \\ Fax +497614520714

\section{(c) 2015 S. Karger GmbH, Freiburg}

$1661-3791 / 15 / 0102-0124 \$ 39.50 / 0$
Prof. Dr. med. Michael Gnant, FACS

Department of Surgery and Comprehensive Cancer Center Vienna Medical University of Vienna

Waehringer Guertel 18-20, 1090 Vienna, Austria

michael.gnant@meduniwien.ac.at 
Table 1. Participants of the St. Gallen/Vienna 2015 Consensus Panel

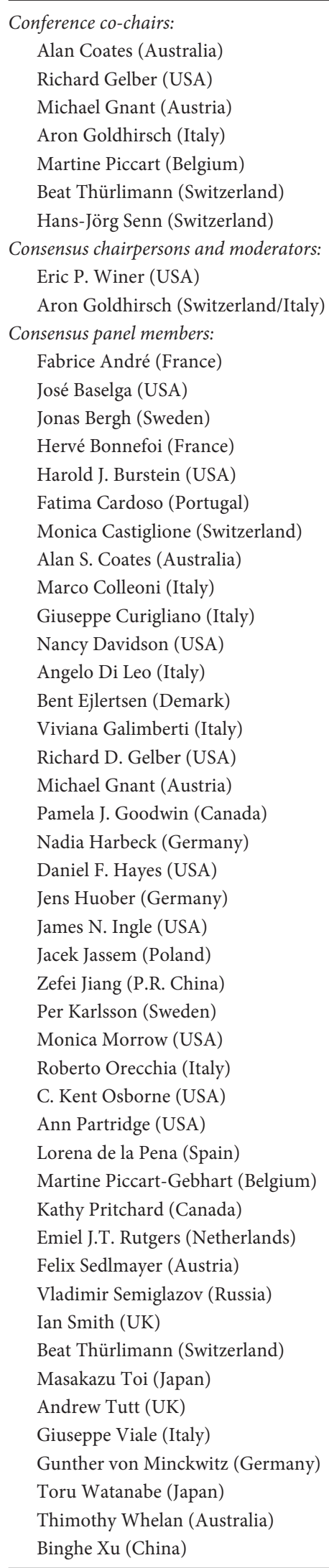

a treatment choice for women with early breast cancer by considering tumor biology and host factors, also balancing benefits and risks of therapies.

The 2015 discussion now finally entirely incorporated a modern differentiation of breast cancer [1] including the concept of intrinsic biological subtypes but also of further molecular characterization of the disease $[2,3]$. Great emphasis was again given to locoregional treatment aspects, with observations that surgery is being further minimized but radiotherapy appears to be becoming more aggressive [4], and surgical tactics do not change much after neoadjuvant therapy. The impact of a rising number of molecular testing tools was assessed, both for judging prognosis of early and late metastasis risk, but also for prediction of therapy benefit. Particularly the benefit of extended endocrine therapy [5] and the current standard of care of endocrine therapies in young patients were extensively covered in light of recent pivotal trial result presentations.

In general, the panelists were asked to cast their vote using 3 possible answers allowed: Yes/No/Abstain. However, due to the complexity of some questions, more options were given in certain instances. 'Abstain' was to be used in the case of insufficient data, no personal expertise on the particular issue, or conflict of interest. After each vote, the answers were summarized in percentages. This report summarizes the original voting questions and resulting percentages of the St. Gallen panel discussion on Saturday March 11, 2015.

\section{Surgery of the Primary Tumor}

Locoregional treatment aspects were again a major topic of this year's St. Gallen/Vienna Consensus: Despite extensive discussions, there were no major changes in technical aspects of primary tumor resection, but it can be noted the 'margin issue' appears now to be resolved and that oncoplastic techniques have found their role the field of breast-conserving surgery. Also, breast-conserving surgery was again confirmed as intended standard of care, also in cases of multifocal (72\% Yes, 14\% No, 14\% Abstain) or multicentric (79\% Yes, $21 \%$ No) disease, provided that clear margins can be achieved and whole-breast radiotherapy is planned.

When asked about the minimum acceptable surgical margin, $92 \%$ of panelists voted for 'no ink on invasive tumor', and $8 \%$ for '1-2 mm' clearance. The panel was clear on whether the margin required should depend on tumor biology (100\% No), should be greater when age is less than 40 years ( $100 \%$ No), should be greater for lobular histology ( $100 \%$ No), and should be greater after neoadjuvant chemotherapy (90\% No, $8 \%$ Yes, $2 \%$ Abstain). A clear majority of panelists felt that margins should not be greater in the presence of extensive intraductal component ( $80 \%$ No, 20\% Yes) and greater for pure ductal carcinoma in situ than for invasive disease ( $80 \%$ No, $20 \%$ Yes).

After downstaging by neoadjuvant chemotherapy, the entire area of the original primary does not need to be resected (89\% Yes, 9\% No, 2\% Abstain). 


\section{Surgery of the Axilla}

Sentinal node (SN) biopsy was once more confirmed as the standard of care [6]. The panel discussions focused on controversial issues of axillary dissection (AD) after positive $\mathrm{SN}$, defining that $\mathrm{AD}$ cannot be omitted in patients with positive $\mathrm{SN}$ if mastectomy without radiotherapy was planned $(100 \%$ No), but the panel was split on whether such omission would be appropriate for patients with mastectomy and radiotherapy ( $52 \%$ Yes, $48 \%$ No). The panel by majority voted that $\mathrm{AD}$ can be omitted after positive $\mathrm{SN}$ in breast conservation situations with radiotherapy using standard tangents (67\% Yes, 33\% No), and high tangents to include the lower axilla (94\% Yes, 3\% No, 2\% Abstain).

After neoadjuvant chemotherapy and successful downstaging of an initially clinically positive axillary node, SN biopsy was considered an appropriate method (90\% Yes, 7\% No, 3\% Abstain); however, the panel felt that $\mathrm{AD}$ should not be avoided if the SN turns out to be positive ( $10 \%$ Yes, $90 \%$ No).

\section{Radiotherapy}

The panel was split over the controversy of whether partial breast irradiation should be used as definitive irradiation for ASTRO (American Society for Radiation Oncology) / ESTRO (European Society for Radiotherapy and Oncology)-'suitable' patients (49\% Yes, 40\% No, 11\% Abstain), but declined this strategy for ASTRO/ESTRO-'cautionary' patients (2\% Yes, 78\% Yes, 22\% Abstain), and did not feel that 'only in the absence of adverse tumor pathology' it would make a difference (22\% Yes, $60 \%$ No, $18 \%$ Yes).

With respect to hypofractionated breast irradiation after breastconserving surgery, the panel felt that this is appropriate for patients aged 50+ without chemotherapy or axillary involvement (89\% Yes, 2\% No, 9\% Abstain), but also for patients younger than 50 years (71\% Yes, 2\% No, 27\% Abstain), with uncertainty about patients with prior chemotherapy or axillary lymph node involvement (51\% Yes, 18\% No, 31\% Abstain).

The panel's vote was unequivocal that after breast-conserving surgery, radiation should include breast only in the case of a negative SN/axilla (100\% Yes). For positive nodes and breast conservation, only $5 \%$ thought that breast-only radiotherapy would be sufficient, with $50 \%$ voting that breast and regional nodes but not internal mammary nodes (IMN) should be irradiated, 30\% voting for radiotherapy of breast, regional nodes and IMN, and 16\% abstaining.

Postmastectomy radiotherapy (PMRT) should be standard of care for T3 (90\% Yes, 10\% No), N+ 1-3 with adverse pathology (87\% Yes, 7\% No, 7\% Abstain), but not for all N+1-3 (32\% Yes, $64 \%$ No, $4 \%$ Abstain) nor $\mathrm{N}+1-3$ at age $<40$ years ( $51 \%$ Yes, $37 \%$ No, $12 \%$ Abstain). PMRT should be given to patients with positive SN but no AD (70\% Yes, $17 \%$ No, $13 \%$ Abstain), but not to pN0 patients after $\mathrm{AD}$ and $<8$ nodes dissected ( $0 \%$ Yes, $95 \%$ No, $5 \%$ Abstain) unless micrometastases were found in the latter situation (71\% Yes, 185 No, 11\% Abstain).
A relative majority of panel members (41\%) found that PMRT should include chest wall and regional nodes but not IMN (32\% Abstain, 11\% Chest wall only, 16\% Chest wall and regional nodes and IMN). Radiotherapy after immediate reconstruction should include the reconstructed breast and nodes in most cases (55\% Yes, $29 \%$ No, 16\% Abstain), with uncertainty about 'only for tumors with adverse pathological features' (37\% Yes, 43\% No, 20\% Abstain).

In general, the panel felt that after neoadjuvant therapy, radiotherapy strategies should follow the stage before neaodjuvant therapy (68\% Yes, $22 \%$ No, $10 \%$ Abstain) rather than after neoadjuvant therapy (24\% Yes, 66\% No, 10\% Abstain).

\section{Pathology, Prognostic, and Predictive Factors}

As in 2013 [7], the panel again agreed that distinction between 'luminal A-like' and 'luminal B-like' (HER2-negative) can be derived from estrogen receptor (ER), progesterone receptor, and Ki-67 status (78\% Yes, 22\% No), and that $\mathrm{Ki}-67$ use requires knowledge of local laboratory values (70.3\% Yes, $13.5 \%$ No). The minimum value of Ki-67 required for 'luminal B-like' was for the majority of the panel $20-29 \%$ (36.4\%). About one fifth (20.5\%) stated that Ki-67 should not be used for this distinction. The majority $(66.7 \%)$ disagreed with the statement that only multigene classifiers (e.g. PAM50, MammaPrint ${ }^{\circledR} /$ Blueprint ${ }^{\circledR}$ (Agendia, Amsterdam, The Netherlands)) can appropriately determine molecular subtype; $59.5 \%$ disagreed with the statement that subtype does not need to be determined since it can be replaced by risk scores derived from multi-gene tests.

The vote was split with regard to the question of whether the extent of lymphocytic infiltration should be reported and used as a prognostic marker in triple-negative breast cancer (TNBC) and HER2-positive disease (45.2\% Yes, 54.8\% No). The majority disagreed with the extent of lymphocytic infiltration to be reported and used as a predictive marker (7.7\% Yes, $89.7 \%$ No).

For the question of whether a chemotherapy decision in patients with ER-positive HER2-negative N0 breast cancer always requires $\mathrm{Ki}-67$ or multi-gene assays, the vote was split (55.6\% Yes, $44.4 \%$ No).

There were 3 questions on all multigene assays, addressing whether they are prognostic for short-term and long-term outcome and whether they predict chemotherapy response.

The Yes answers are summarized in table 2.

\section{Endocrine Therapy}

\section{Premenopausal}

Surely affected by the recent data on ovarian function suppression (OFS) as adjuvant treatment option in premenopausal patients, a substantial part of the discussion was focused on this topic $[8,9]$. Thus, the session on endocrine treatment began with a discussion of 2 cases. In case 1, recommendation of standard adjuvant 
Table 2. 'Yes' votes for multigenomic assays

\begin{tabular}{llllll}
\hline & $\begin{array}{l}\text { Recurrence } \\
\text { score }\end{array}$ & $\begin{array}{l}\text { Mamma } \\
\text { print }\end{array}$ & Prosigna & $\begin{array}{l}\text { Endo- } \\
\text { predict }\end{array}$ & $\begin{array}{l}\text { Breast cancer } \\
\text { index }\end{array}$ \\
\hline $\begin{array}{c}\text { Short-term } \\
\text { outcome }\end{array}$ & 85 & 81 & 93 & 70 & 58 \\
$\begin{array}{c}\text { Long-term } \\
\text { outcome }\end{array}$ & 44 & 15 & 63 & 38 & 31 \\
$\begin{array}{c}\text { Chemotherapy } \\
\text { response }\end{array}$ & 81 & 35 & 38 & 24 & 10 \\
\hline
\end{tabular}

endocrine therapy for a 42-year-old patient with an intermediate grade (G2), node-negative, steroid hormone receptor-positive breast cancer, having not received adjuvant chemotherapy, was discussed. The majority of the panelists $(85.0 \%)$ voted for treatment with tamoxifen alone, $12.5 \%$ for OFS plus tamoxifen. In a second case, the panelists were asked to vote on adjuvant endocrine treatment of a 34-year-old patient who remained premenopausal after adjuvant chemotherapy for a p 11 , grade 3 , node-positive steroid hormone receptor-positive breast cancer. The majority would treat her with OFS and exemestane $(69.8 \%)$, a minority (23.3\%) with OFS and tamoxifen. Only a few panelists voted for tamoxifen $(2.3 \%)$ alone or abstained.

Then, general recommendations of the indication of OFS were discussed. Most panelists voted for considering age $\leq 35$ years (81\%), premenopausal estrogen levels after chemotherapy (73.7\%), and high tumor load (4 or more axillary lymph nodes; $89.7 \%$ ) as arguments in favor of OFS. Unfavorable biology, i.e. grade 3 $(55.9 \%$ Yes, $38.2 \%$ No) or adverse result of multi-gene test $(60 \%$ Yes, $24.4 \%$ No), was less clearly judged as argument in favor of OFS. Then, more explicitly, the preference of OFS plus an aromatase inhibitor (AI) rather than OFS and tamoxifen was discussed. The strongest arguments in favor of using AI were grade 3 (92.5\%) and high tumor load (4 or more axillary lymph nodes; $92.5 \%)$, followed by adverse result of multi-gene test (65.8\%) and age $\leq 35$ years $(59.4 \%$ Yes, $37.5 \%$ No). Some more panelists felt that premenopausal estrogen level after adjuvant chemotherapy should not be an argument in favor of the use of $\mathrm{AI}$ in combination with OFS (51.2\% Yes, $43.9 \%$ No). In order to give a clear recommendation as to the preferred combination if OFS seems to be necessary, the panel was also asked for general preference between tamoxifen and AI; the majority (58.5\%) voted for recommending the combination of AI with OFS, only $36.6 \%$ considered tamoxifen plus OFS as treatment of choice.

\section{Postmenopausal}

Tamoxifen was still considered as adequate treatment in some postmenopausal patients (97.6\%); however, clear factors arguing for inclusion of an AI were high tumor load defined by 4 or more tumor-infiltrated axillary lymph nodes (97.6\%), or unfavorable biology defined by grade 3 or high Ki-67 (97.7\%), or HER2 positivity (71.1\%). Age (> 60 years) was not considered as an argument for $\mathrm{AI}$ inclusion (31\% Yes, $69 \%$ No).

With regard to the sequence of $\mathrm{AI}$ and tamoxifen, a slight majority declined to start AI upfront in all patients (47.5\% Yes, 52.5\%
No); however, in patients at higher risk, upfront AI was clearly regarded as the preferred mode of therapy (95.5\% Yes). According to the vote of the panelists (75.0\%), upfront AI can be switched to tamoxifen after 2 years.

\section{Duration}

Recent publications studied prolongation of the adjuvant endocrine treatment exceeding the traditional duration of 5 years, and the results suggested advantages in some patients [10-12]. In a detailed discussion, votes on several scenarios were performed.

In patients with node-positive disease who are disease-free after 5 years of tamoxifen, a vast majority of the panelists would recommend continuing AI / AI/OFS or tamoxifen up to 10 years (premenopausal 100\%, postmenopausal 95.2\%). For patients with node-negative disease, most panelists voted against prolongation (premenopausal 74.4\%, postmenopausal $80.5 \%$ ). However, in patients with grade 3 or high Ki-67, the majority would advise prolongation of endocrine therapy (premenopausal 73.8\%, postmenopausal $76.7 \%)$.

Finally, for those postmenopausal patients who were premenopausal at baseline, a majority of panelists (66.7\%) would recommend a prolongation of endocrine therapy, presumably triggered by the subgroup analysis of the MA.17 trial that showed particular benefit of letrozole after 5 years tamoxifen in these patients [12].

With regard to the details of the specific treatment in the case of an indication for endocrine therapy beyond the first 5 years, the discussion had the following results: After 5 years of adjuvant therapy involving switch from tamoxifen to an AI (therefore assuming postmenopausal status at the 5-year time point and reasonable tolerance to endocrine therapy), most panelists voted to recommend continuation of AI to a cumulative total of 5 years (75.0\%). A further 5 years of pure tamoxifen or AI treatment was supported only by a minority (tamoxifen $39.4 \%$, AI $31.4 \%$ ). Finally, the voting was focused on treatment recommendations after 5 years of straight AI adjuvant therapy; although some panelists (40.9\% Yes) voted in favor of further prolongation of adjuvant endocrine therapy (tamoxifen or AI), the majority thought that 5 years of AI would be sufficient and voted not to recommend further endocrine therapy (tamoxifen 63.4\% No; AI 57.1\% No). However, being asked for a specific recommendation in a patient with moderate risk of recurrence and tolerating the endocrine treatment, the answers of the panelists were even less clear-cut between 3-5 years of tamoxifen (27\%), 3-5 years of an AI (37.8\%), and no further endocrine therapy $(29.7 \%)$. 
The voting on the optimal duration of OFS in a premenopausal woman for whom this treatment is indicated was rather unambiguous; most panelist (56.7\%) recommended 5 years or even life-long (3.3\%), a minority (16.7\%) voted for a shorter period of $2-3$ years, and a substantial proportion abstained (23.3\%).

\section{Chemotherapy}

The panel voted that the following factors constituted relative indications for the inclusion of adjuvant chemotherapy: Histological grade 3 tumor ( $97.4 \%$ Yes); any positive node (38.7\% Yes); 4 or more positive nodes (95.1\% Yes); high Ki-67 (75.0\% Yes); age < 35 years ( $41.7 \%$ Yes); extensive lymphovascular invasion (67.6\% Yes); low hormone receptor staining (81.1\% Yes).

The majority agreed that luminal A phenotype needs to be considered as less responsive to chemotherapy ( $88.1 \%$ Yes). Regarding risk factors indicating use of chemotherapy in luminal $\mathrm{A}$, the panel voted for the following factors: Tumor size (36.4\% Yes, $63.2 \%$ abstained when asked about a minimum size); lymphovascular invasion (28.6\% Yes); $1-3$ involved nodes (34.9\% Yes); $\geq 4$ involved nodes $(91.1 \%$ Yes).

The majority did not think that in IHC-luminal B-like tumors chemotherapy should be recommended in all patients (22.0\% Yes), but rather only in patients with other indicators of increased risk (87.5\% Yes). In patients with luminal B-like disease and a low Oncotype Dx ${ }^{\circledR}$ (Genomic Health, Inc. Redwood City, CA, USA) score ( $94.9 \%$ Yes) but not an intermediate score (36.4\% Yes), chemotherapy may be omitted. Chemotherapy may also be omitted in patients with a low-risk Mammaprint result $(72.1 \%$ Yes), a low PAM50 risk of recurrence score (82.5\% Yes), and a low-risk EndoPredict ${ }^{\circledR}$ (Sividon, Köln, Germany) result (69.6\% Yes).

In luminal B (HER2-negative) tumors, chemotherapy should include an anthracycline (83.3\% Yes) and a taxane (76.9\% Yes). The panel stated that 6 cycles of the same chemotherapy, such as $6 \times$ AC (cyclophosphamide, doxorubicin) or 6× FEC (fluorouracil, epirubicin, cyclophosphamide), should not be given anymore ( $21.6 \%$ Yes). $57.1 \%$ believed that there was a high-risk group for which dose-dense chemotherapy should be preferred.

In TNBC, chemotherapy should contain anthracyclines and taxanes $(92.3 \%$ Yes). Only $7.1 \%$ believed a platinum-based regimen should be considered in all TNBC patients. The majority (57.9\%) would consider a platinum-based regimen only in known BRCA mutation carriers. Nevertheless, the majority (75\%) also thought that a standard anthracycline- and taxane-containing regimen is still acceptable in TNBC with BRCA mutation. Only 45\% thought that dose-dense chemotherapy requiring growth factor support should be preferred in TNBC.

In HER2-positive disease, 97\% believed chemotherapy should always be given to patients with stage 2 disease who require antiHER2 therapy. $88.9 \%$ believed that this chemotherapy should contain anthracyclines, and $97.2 \%$ believed that it should contain taxanes. 97.3\% agreed that the anti-HER2 therapy should start concurrent with the taxane. In patients with HER2 positivity ac- cording to American Society of Clinical Oncology (ASCO)/College of American Pathologists (CAP) guidelines, the panel voted for anti-HER2 therapy in stage 1 disease in T1a (20.7\% Yes), T1b (81.4\% Yes), and T1c (100\%). Here, only $57.9 \%$ thought that the chemotherapy should contain anthracyclines (57.9\%), and $86.5 \%$ agreed that paclitaxel and trastuzumab would be a reasonable option.

For patients requiring adjuvant anti-HER2 therapy for a T2 tumor with 4 involved nodes, only $21.4 \%$ thought that therapy should include both trastuzumab and pertuzumab, and only $8 \%$ thought that it should contain both trastuzumab and lapatinib.

Among neoadjuvant therapy choices for HER2-positive stage II disease, $23.1 \%$ considered taxane, trastuzumab and pertuzumab, and $56.4 \%$ anthracycline $\rightarrow$ taxane + anti-HER2 an acceptable regimen. If asked whether taxane, trastuzumab, and pertuzumab is an acceptable neoadjuvant regimen for HER2-positive stage II disease, $73.1 \%$ agreed and only $39.3 \%$ considered platinum, taxane, trastuzumab \pm pertuzumab as an acceptable regimen. A non-taxane regimen containing platinum, trastuzumab \pm pertuzumab only got $2.9 \%$ Yes votes whereas anthracycline $\rightarrow$ taxane and anti-HER2 got 97.2\% Yes votes.

For stage II TNBC, only $17.2 \%$ thought that the preferred neoadjuvant regimen should include a high-dose alkylating agent, $25 \%$ that it should include a platinum compound, and $94.7 \%$ that it should contain anthracycline $\rightarrow$ taxane. Only $22.9 \%$ voted for nab-paclitaxel $\rightarrow$ EC (epirubicin, cyclophosphamide), and $25.7 \%$ for an anthracycline regimen with alkylating agents (e.g. classical CMF (cyclophosphamide, methotrexate, fluorouracil)) in this context.

$73.5 \%$ believed that neoadjuvant cytotoxic therapy should be discussed as an option in patients with 'luminal A-like' tumors only if conservative surgery would not otherwise be feasible. Yet, only $16 \%$ believed neoadjuvant cytotoxic therapy should be discussed as an option in patients with 'luminal A-like' tumors.

$87.9 \%$ considered neoadjuvant endocrine therapy without cytotoxics a reasonable option for postmenopausal patients with endocrine responsive disease. $42.9 \%$ considered $4-8$ months as an optimal therapy duration, and $42.9 \%$ favored therapy until best response.

\section{Bisphosphonates}

For the first time, a majority of the panel voted that adjuvant bisphosphonates, such as zoledronic acid every 6 months or oral clodronate, are indicated during adjuvant endocrine therapy for postmenopausal patients with endocrine responsive disease ( $58 \%$ Yes, $42 \%$ No). The panel was split on premenopausal patients receiving luteinizing hormone-releasing hormone (LHRH) plus tamoxifen (44\% Yes, 56\% No), but clear that bisphosphonates should not be used for premenopausal patients not receiving LHRH (5\% Yes, 95\% No) and that denosumab should not be a substitute for bisphosphonates (4\% Yes, $89 \%$ No, 7\% Abstain). 


\section{Special Issues and Patient Populations}

For elderly patients, the panel was very clear that for standard chemotherapy there is no absolute age limit, rather disease factors, co-morbidities, life expectancy, and patient preferences shall be factors in the decision (87\%). As for radiotherapy in elderly patients, $30 \%$ of panel members felt that it should be omitted over the age of 70 years after breast conservation in ER-positive tumors, but $56 \%$ felt that the same multifactorial approach as described above should be taken.

For young patients, a majority of the panel felt that all patients younger than 40 years should be tested for BRCA 1 and 2 (73\% Yes, 24\% No, 3\% Abstain). For patients with TNBC, BRCA testing should be done in all patients $<40$ years of age $(91 \%$ Yes, $6 \%$ No, $3 \%$ Abstain) but not all patients $<60$ years of age (49\% Yes, $49 \%$ No, 2\% Abstain).

The majority of the panel felt that discovery of a BRCA 1 or 2 mutation influences the treatment strategy with respect to the locoregional (78\% Yes, $22 \%$ No) or neoadjuvant (66\% Yes, 35\% No) but not adjuvant strategy (29\% Yes, $66 \%$ No, 5\% Abstain).

Only a minority of the panel felt that all breast cancer patients ( $11 \%$ Yes), or patients < 40 years of age $(41 \%$ Yes, $50 \%$ No) should be tested for high-risk mutations of other genes. However, such testing should be done for patients with a strong family history (95\% Yes), or age $<35$ years at diagnosis $(89 \%$ Yes), or age $<50$ years in ER-/HER2-negative tumors ( $70 \%$ Yes, 30\% No). The panel was split on this question in patients with basal-like tumors $(49 \%$ Yes, 46\% No, 5\% Abstain).

The panel was clear that fertility preservation should be offered to women $<40$ years of age ( $88 \%$ Yes, $10 \%$ No, $2 \%$ Abstain), as well as OFS during chemotherapy in receptor-negative disease (79\% Yes, 18\% No, 3\% Abstain).

For breast cancer diagnosed during pregnancy, the panel voted that premature delivery should be avoided if possible (89\% Yes, $4 \%$ No, $7 \%$ Abstain), breast conservation is a suitable option (89\% Yes, $3 \%$ No, $8 \%$ Abstain), lymphoscintigraphy and SN biopsy are safe (65\% Yes, 29\% No, 6\% Abstain), and anti-HER2 therapy should be delayed until after delivery (87\% Yes, $8 \%$ No, 5\% Abstain). However, the panel was unclear about immediate postmastectomy reconstruction as an appropriate option (53\% Yes, 39\% No, 10\% Abstain).

With respect to attempting pregnancy after breast cancer, the panel felt that it is reasonable to interrupt endocrine therapy after
18-30 months (61\% Yes, 30\% No, 9\% Abstain) and only in the absence of high-risk factors (61\% Yes, $28 \%$ No, $11 \%$ Abstain), but not at any time during endocrine therapy (26\% Yes, $68 \%$ No, $6 \%$ Abstain).

For endocrine-responsive male breast cancer, the panel felt that tamoxifen should be recommended, but not AI (29\% Yes, 58\% No, $13 \%$ Abstain) nor AI + LHRH (30\% Yes, 67\% No, 3\% Abstain).

With respect to diet and exercise after breast cancer, the panel did not recommend specific dietary advice (40\% Yes, 58\% No, $2 \%$ Abstain), but favored vitamin D supplementation for vitamin Ddeficient patients (57\% Yes, 34\% No, 9\% Abstain) and exercise regimens as standard of care ( $77 \%$ Yes, $23 \%$ No), and recommended weight loss/avoidance of weight gain (88\% Yes, 8\% No, 5\% Abstain).

\section{Conclusion}

In summary, St. Gallen/Vienna 2015 was a highly successful conference. International attendance increased as compared to the previous meeting, and the panel conquered a record number of almost 200 questions, likely because of excellent iterative preparation of the questions in the months and weeks preceding the conference. Overall, recommendations were further moving toward the 'biology of the disease', with increasing attention to host factors and special patient populations. This reflects the way the field is moving forward in terms of gathering knowledge, and will be helpful in many difficult or conflicting treatment decision situations. Based on experience and opinion by distinguished international experts rather than on systematic evidence, the results may differ from national [13] or international guidelines, but will still be an important source of advice for many physicians and patients.

\section{Disclosure Statement}

Dr. Gnant reports receiving grants from: Sanovi Aventis, Novartis, Roche, GlaxoSmithKline, Pfizer and Smith Medical, and personal fees from: Novartis, Roche, GlaxoSmithKline, AstraZeneca, Nanostring Technologies and Accelsiors. Dr. Thomssen reports receiving honoraria for lectures and advisory board membership from Amgen, Astra-Zeneca, Celgene, Genomic Health, Nanostring, Novartis, Pfizer, Roche, and TEVA. Dr. Harbeck reports receiving honoraria for lectures and advisory board membership from Celgene, Genomic Health, Nanostring, Novartis, Roche.

\section{References}

1 Gnant M: Modern therapeutic concepts of early breast cancer. Breast Care 2014;9:85-86.

2 Gluz O, Hofmann D, Würstlein R, Liedtke C, Nitz U, Harbeck N: Genomic profiling in luminal breast cancer. Breast Care 2013;8:414-422.

3 Liedtke C, Bernemann C, Kiesel L, Rody A: Genomic profiling in triple-negative breast cancer. Breast Care 2013;8:408-413.
4 Sanuki N, Takeda A, Amemiya A, Ofuchi T, Ono M, Ogata H, Yamagami R, Hatayama J, Eriguchi T, Kunieda E: Axillary irradiation with high tangent fields for clinically node-negative breast cancer: can 3-D conformal radiotherapy with a field-in-field technique better control the axilla? Breast Care 2013;8:362-367.

5 Knauer M, Filipits M, Dubsky P: Late recurrences in early breast cancer: for whom and how long is endocrine therapy beneficial? Breast Care 2014;9:97-100.
6 Reimer T, Hartmann S, Stachs A, Gerber B: Local treatment of the axilla in early breast cancer: concepts from the National Surgical Adjuvant Breast and Bowel Project B-04 to the Planned Intergroup Sentinel Mamma Trial. Breast Care 2014;9:87-95.

7 Goldhirsch A, Winer EP, Coates AS, Gelber RD, Piccart-Gebhart M, Thürlimann B, Senn HJ; Panel members: Personalizing the treatment of women with early breast cancer: highlights of the St Gallen International Expert Consensus on the Primary Therapy of Early Breast Cancer 2013. Ann Oncol. 2013;24:2206-2223. 
8 Francis PA, Regan MM, Fleming GF, Láng I, Ciruelos E, Bellet M, Bonnefoi HR, Climent MA, Da Prada GA, Burstein HJ, Martino S, Davidson NE, Geyer CE Jr, Walley BA, Coleman R, Kerbrat P, Buchholz S, Ingle JN, Winer EP, Rabaglio-Poretti M, Maibach R, Ruepp B, Giobbie-Hurder A, Price KN, Colleoni M, Viale G, Coates AS, Goldhirsch A, Gelber RD; SOFT Investigators, International Breast Cancer Study Group: Adjuvant ovarian suppression in premenopausal breast cancer. N Engl J Med 2015;372:436-446.

9 Pagani O, Regan MM, Walley BA, Fleming GF, Colleoni M, Láng I, Gomez HL, Tondini C, Burstein HJ, Perez EA, Ciruelos E, Stearns V, Bonnefoi HR, Martino S, Geyer CE Jr, Pinotti G, Puglisi F, Crivellari D, Ruhstaller T, Winer EP, Rabaglio-Poretti M, Maibach R, Ruepp B, Giobbie-Hurder A, Price KN, Bernhard J, Luo W, Ribi K, Viale G, Coates AS, Gelber RD, Goldhirsch A, Francis PA; TEXT and SOFT Investigators, International Breast Cancer Study Group: Adjuvant exemestane with ovarian suppression in premenopausal breast cancer. N Engl J Med 2014;371:107-118.
10 Davies C, Pan H, Godwin J, Gray R, Arriagada R, Raina V, Abraham M, Medeiros Alencar VH, Badran A, Bonfill X, Bradbury J, Clarke M, Collins R, Davis SR, Delmestri A, Forbes JF, Haddad P, Hou MF, Inbar M, Khaled H, Kielanowska J, Kwan WH, Mathew BS, Mittra I, Müller B, Nicolucci A, Peralta O, Pernas F, Petruzelka L, Pienkowski T, Radhika R, Rajan B, Rubach MT, Tort S, Urrútia G, Valentini M, Wang Y, Peto R; Adjuvant Tamoxifen: Longer Against Shorter (ATLAS) Collaborative Group: Long-term effects of continuing adjuvant tamoxifen to 10 years versus stopping at 5 years after diagnosis of oestrogen receptorpositive breast cancer: ATLAS, a randomised trial. Lancet 2013;381:805-816.

11 Gray RG, Rea D, Handley K, Bowden SJ, Perry, Earl HM, Poole CJ, Bates T, Chetiyawardana S, Dewar JA, Fernando IN, Grieve R, Nicoll J, Rayter Z, Robinson A, Salman A, Yarnold J, Bathers S, Marshall A, Lee M; on behalf of the aTTom Collaborative Group: aTTom: long-term effects of continuing adjuvant tamoxifen to 10 years versus stopping at 5 years in 6,953 women with early breast cancer. J Clin Oncol 2013;31:abstr 5.
12 Ingle JN, Tu D, Pater JL, Muss HB, Martino S, Robert NJ, Piccart MJ, Castiglione M, Shepherd LE, Pritchard KI, Livingston RB, Davidson NE, Norton L, Perez EA, Abrams JS, Cameron DA, Palmer MJ, Goss PE: Intentto-treat analysis of the placebo-controlled trial of letrozole for extended adjuvant therapy in early breast cancer: NCIC CTG MA.17. Ann Oncol 2008;19:877-882.

13 Liedtke C, Thill M, Hanf V, Schütz F; on behalf of the AGO Breast Committee: AGO recommendations for the Diagnosis and Treatment of Patients with Early Breast Cancer: Update 2014. Breast Care 2014;9:189200. 